\title{
The next personalized medicine evolution in orthopedics: how diagnosing and treating scoliosis are about to change
}

\author{
"...clear conceptual gaps exist in our understanding of scoliosis \\ pathogenesis that result in barriers to move this field forward."
}

First draft submitted: 7 November 2016; Accepted for publication: 12 December 2016; Published online: 19 January 2017

Keywords: bracing $\bullet$ diagnostic $\bullet$ endophenotypes $\bullet$ genomics $\bullet$ idiopathic scoliosis

- metabolomics $\bullet$ minimally invasive surgery $\bullet$ pharmacotherapies

\begin{abstract}
Adolescent idiopathic scoliosis (AIS) is a common complex multifactorial disease and one of the most prevalent childhood deformities worldwide [1,2]. On average, AIS affects $4 \%$ of the global pediatric population [3]. It represents a serious and chronic health condition affecting individuals all throughout their lives. More than 7 million patients in the USA are diagnosed with scoliosis (over 350,000 in Canada). Most are diagnosed with scoliosis between the ages of 10 and 15 years, and one out of every six children will have a progressive curve that requires active treatment. Due to its persistently idiopathic nature, the standard care for AIS has not significantly changed in decades. Current patients are treated by observation, bracing and, as a last resort, spinal-fusion surgery. Because of the patients' discomfort and risk, the application of current treatments is delayed until a significant deformity or progression is detected. This results in suboptimal treatment, significant psychological sequelae and heavy economic burdens for the families and healthcare systems ( US\$2.7 billion in USA annually).
\end{abstract}

\section{Decoding idiopathic scoliosis: why are we not there yet?}

Although advancements in genomic technologies are transforming many aspects of how we conduct genetic and genomic studies, success thus far has been limited in deciphering complex diseases like AIS and translating discoveries to clinical applications for health improvement and reduction of economic burdens associated with current treatments. This is largely due to apparent genetic heterogeneity of AIS, the fact that a limited number of patients exhibit a spinal deformity progressing to the point of requiring a corrective surgery and a lack of consensus about our understanding of the AIS pathophysiology [4-6]. Although a genetic basis is acknowledged from familial studies, the mode of inheritance appears to be heterogeneous. Reports of AIS inheritance include autosomal dominant, autosomal recessive and multifactorial (reviewed by Gorman et al. [7]). The majority of genetic studies for AIS prior to 2010 are candidate gene based, with candidates selected from hypotheses generated by clinical observations. Most of these genes remain unconfirmed or have mixed published results, depending on the design of the study and/or populations tested (reviewed by Gorman et al. [5]). Nevertheless, clear conceptual gaps exist in our understanding of scoliosis pathogenesis that result in barriers to move this field forward. Firstly, scoliosis is not per se a disease but rather a phenotypic trait present and common to several medical conditions. Secondly, there is presently no widely accepted model for AIS that could explain scoliosis onset, the multiplicity of associated neuroendocrine abnormalities or the cause(s) of spinal deformity progression. While the mechanisms underpinning these disturbances are unknown, several lines

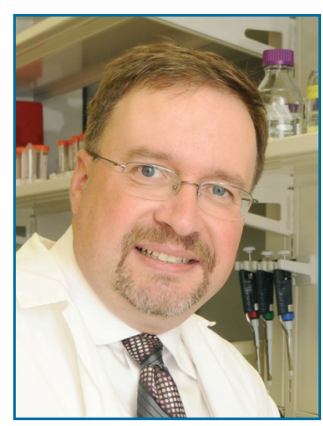

Alain Moreau

Viscogliosi Laboratory in Molecular Genetics of Musculoskeletal Diseases,

Sainte-Justine University Hospital Research Center, Montréal, 3175 Cote-SteCatherine Road, Montreal, Québec, H3T 1C5, Canada and

Department of Stomatology, Faculty of Dentistry,

Université de Montréal,

Montréal, Québec, H3T 1J4, Canada

and

Department of Biochemistry

\& Molecular Medicine,

Faculty of Medicine,

Université de Montréal,

Montréal, Québec, H3T 1J4,

Canada

alain.moreau@

recherche-ste-justine.qc.ca 
of evidence suggest that Gi-coupled receptor signaling dysfunction could be at the source of these abnormalities [8]. Thirdly, despite the fact that scoliosis affects millions of individuals worldwide, the scoliosis research community is very small and too often stratified by barely intersecting themes. Furthermore, the general view by many clinicians and researchers that AIS is a complex and multifactorial disease gives rise to diverse hypotheses and opinions regarding its pathophysiology, which are rarely challenged or verified.

$$
\begin{aligned}
& \text { "We should not be justified in devoting further time } \\
& \text { to opinions, which are not supported by any serious } \\
& \text { experiment." } \\
& \text { - Louis Pasteur (1822-1895) }
\end{aligned}
$$

\section{The promises of the genome-wide association study \& whole-exome sequencing era}

Considering the lack of success from AIS hypothesisdriven genetic studies and the prevalence of the disease, the application of genome-wide association study (GWAS) to AIS was logical, with the expectation that a number of modest effect variants associated to predisposition and/or severity would be identified. The first AIS GWAS was conducted by Sharma et al. [9] who surveyed 419 trio-families (affected children and their parents) in Utah, to generate a list of 100 significantly associated SNPs that were then combined with three independent replication studies for a total of 3431 individuals. The most significant SNP (rs10510181) had an odds ratio (OR): 1.37 (95\% CI: $\left.1.20-1.58 ; \mathrm{p}=8.22 \times 10^{-7}\right)$. The results suggested that $C H L 1$, a member of the $L 1$ gene family of neural cell adhesion molecules, might be associated with susceptibility. The authors then surveyed variants significantly associated with other genes involved in the axon guidance pathway: $\operatorname{DSCAM}\left(\mathrm{p}=2.26 \times 10^{-5}\right.$ for rs2222973) and CNTNAP2 ( $\mathrm{p}=6.20 \times 10^{-5}$ for rs11770843) but concluded that larger cohorts are necessary to verify their findings and identify additional susceptibility loci. Another study using a Han Chinese population did not replicate the association between CHL1 and AIS [10], suggesting that either the risk is population specific or is a type I error. Takahashi et al. [11] used 1376 Japanese females with AIS and 11,297 female controls to demonstrate an association to three variants (combined $\mathrm{p}=1.24 \times 10^{-19}$; OR: 1.56$)$ near $L B X 1$, a transcription factor required for the development of inhibitory interneurons in the dorsal horn of the spinal cord, as well as migration and development of hypaxial muscle precursor cells for limb muscles. The association was independently replicated in two Han Chinese populations [12]. Of note, Kou et al. [13] demonstrated a modest association $\left(\mathrm{p}=2.25 \times 10^{-10}\right.$; OR: 1.28$)$ between a variant in
GPR126 and 1819 Japanese AIS cases (vs 25,939 controls). In summary, these recent GWAS suggest two, possibly three, genes that pose a minor risk for AIS susceptibility.

Although GWAS have been successful in identifying possible risk alleles for complex diseases such as multiple sclerosis and autoimmune diseases, for other common complex diseases like autism, schizophrenia and inflammatory bowel disease, there is evidence implicating rare variants in their onsets (reviewed in [14]). Considering the low number of risk alleles associated with AIS, rare variants are an important consideration. Rare variants are more likely to be mutations with functional consequences and thus are more likely disease causing $[15,16]$. However, there are some important limitations to consider with GWAS and whole-exome sequencing (WES) approaches. Indeed, the effectiveness of a study is subject to several factors such as sample size, OR, allele frequency, threshold of significance and the performance of the commercial arrays in diverse populations.

\section{Bench-to-bedside medicine: lessons learned from the Scoliscore}

In 2010, a first genetic test using 53 SNPs from a saliva sample (ScoliScore) was released on the market [17]. Unfortunately, three distinct independent studies were unable to replicate the initial findings published by Ward et al. in 2010 [17]. Indeed, ScoliScore markers could not predict AIS curve progression in Japanese, Han Chinese and French-Canadian populations [18-20]. Notwithstanding the initial interest that accompanied the launch of ScoliScore, its clinical utility and validity is increasingly challenged by clinicians due to its immediate limitations [21]. Consistently, it targets only symptomatic white Caucasian girls between the ages of 9 and 13 years, and relies largely on the parameters of age and curve magnitude at the time of the test to stratify patients. The argument was raised that one could potentially risk-stratify patients on the basis of these two clinical parameters alone. Therefore, the ScoliScore is not considered a pure genetic test and has limited to no value as a screening test, because those that have scoliosis already know it. Additionally, many subjects fell into an intermediate score range, a value that was useless in determining the risk of scoliosis progression.

The clinical utility of any predictive test is the likelihood that its use to manage the disease will significantly improve health-related outcomes. Given the clinical heterogeneity of idiopathic scoliosis and its multifactorial nature, it is quite unlikely that a genetic test will be adopted without robust in-field testing by independent groups, using a multicenter protocol among different populations. In this clinical scenario, early detection of scoliosis is not only critical for successful and less inva- 
sive clinical outcomes, but broadens the range of treatment options (e.g., minimally invasive surgeries) and increases effectiveness through personalized medicine.

\section{Beyond genomics: the unexplored scoliosis frontiers}

One of the major challenges in effective treatment of various disorders lies in the timing of diagnosis and timing of evolution of the disorder per se. Although major advances in medicine have been achieved over the last few decades, very often targets selected for treatment are based either on pathogenesis or protracted evolution of the medical condition; these treatments tackle mechanisms distal to the ongoing pathophysiologic process. This claim is true for AIS and a variety of diseases including cancer, chronic inflammatory disorders and other rare and complex diseases. Research on biomarkers has helped to highlight their potential to predict the clinical phenotype, the activity of the disease and its severity, remission and relapse phases, response to treatment (e.g., bracing) and disease staging over time. Given the clinical heterogeneity of the disease, gender differences (higher prevalence in girls) and potential crosstalk with undefined environmental factors, more integrated and comprehensive approaches are required. It is conceivable that search for specific biomarkers or their validation be tied as closely as possible to specific functional assays. By functional assays, we mean deliberate interventions that produce a measurable change in symptomatology, such as controlled exercise testing can be used both to measure physiological parameters but also to generate a reproducible experimental stimulus. In that context, metabolomics, proteomics and transcriptomics approaches could be more useful than genomics to reveal biomarkers correlated with clear changes in symptomatology through such an intervention in a controlled experiment. For instance, in AIS patients involved in bracing trial, there will be both responders and nonresponders. Indeed, the factors underlying brace response remain unknown and the mechanisms by which biomechanical cues affect cellular functions are poorly understood. The combination of different 'omic' approaches could contribute to better understand the differential processing and interpretation of these signals between affected and healthy individuals, which remains a largely unexplored frontier.

\section{The future of scoliosis treatment}

The future of scoliosis treatment lies in early molecular diagnosis and biopharmacological treatments of the growth abnormalities that lead to spinal curvature. Although several genes are known to cause spinal deformities in mice and zebrafish, there has been limited progress in delineating the molecular basis underlying idiopathic scoliosis in humans. Therefore, it is conceivable that most of these genes must connect with a common downstream pathogenic pathway. The search for such a converging mechanism is most likely the key to solve the scoliosis enigma. By targeting the causes underlying scoliosis development, we are at the brink of unlocking the secrets of predicting which child will have a progressive curve, and more importantly, pave the way for the development of pharmacological treatments for the scoliosis prevention in at-risk pediatric populations and mitigating curve progression in symptomatic children.

\section{Financial \& competing interests disclosure}

A Moreau's research program on idiopathic scoliosis has led to several patent applications on behalf of Sainte-Justine University Hospital (CHU Sainte-Justine) and 29 patents have been issued and owned by CHU Sainte-Justine, which has signed a licensing agreement with Fourth Dimension Spine LLC (NYC, USA). The author has no other relevant affiliations or financial involvement with any organization or entity with a financial interest in or financial conflict with the subject matter or materials discussed in the manuscript apart from those disclosed.

No writing assistance was utilized in the production of this manuscript.

\section{Open access}

This work is licensed under the Attribution-NonCommercialNoDerivatives 4.0 Unported License. To view a copy of this license, visit http://creativecommons.org/licenses/by-nc-nd/4.0/

\section{References}

1 Cheng JC, Tang NL, Yeung HY et al. Genetic association of complex traits: using idiopathic scoliosis as an example. Clin. Orthop. Rel. Res. 462, 38-44 (2007).

2 Wise CA, Gao X, Shoemaker S et al. Understanding genetic factors in idiopathic scoliosis, a complex disease of childhood. Curr. Genomics. 9(1), 51-59 (2008).

3 Scoliosis Research Society. Morbidity and Mortality Committee Annual Report (1997). www.srs.org/
4 Lombardi G, Akoume MY, Colombini A et al. Biochemistry of adolescent idiopathic scoliosis. Adv. Clin. Chem. 54, 165-182 (2011).

5 Gorman KF, Julien C, Moreau A. The genetic epidemiology of idiopathic scoliosis. Eur. Spine. J. 21(10), 1905-1919 (2012).

6 Cheng JC, Castelein RM, Chu WM et al. Adolescent idiopathic scoliosis. Nat. Rev. Dis. Primers. (1), 1-20 (2015).

7 Gorman KF, Julien C, Oliazadeh N et al. Genetics of idiopathic scoliosis. In: Encyclopedia of Life Sciences. Copper DN (Ed.). Wiley, UK (2014). 
8 Julien C, Gorman KF, Akoume MY et al. Towards a comprehensive diagnostic assay for scoliosis. Per. Med. 10(1), 97-103 (2013)

9 Sharma S, Gao X, Londono D et al. Genome-wide association studies of adolescent idiopathic scoliosis suggest candidate susceptibility genes. Hum. Mol. Genet. 20(7), 1456-1466 (2011).

10 Qiu XS, Lv F, Zhu ZZ et al. Lack of association between the CHL1 gene and adolescent idiopathic scoliosis susceptibility in Han Chinese: a case-control study. BMC Musculoskelet. Disord. 15, 38 (2014).

11 Takahashi Y, Kou I, Takahashi A et al. A genome-wide association study identifies common variants near $L B X 1$ associated with adolescent idiopathic scoliosis. Nat. Genet. 43, 1237-1240 (2011).

12 Fan YH, Song YQ, Chan D et al. SNP rs11190870 near $L B X 1$ is associated with adolescent idiopathic scoliosis in southern Chinese. J. Hum. Genet. 57, 244-246 (2012).

13 Kou I, Takahashi Y, Johnson TA et al. Genetic variants in GPR126 are associated with adolescent idiopathic scoliosis. Nat. Genet. 45, 676-679 (2013).

14 Casals F, Idaghdour Y, Hussin J et al. Next-generation sequencing approaches for genetic mapping of complex diseases. J. Neuroimmunol. 248(1-2), 10-22 (2012).

15 Marth GT, Yu F, Indap AR et al. The functional spectrum of low-frequency coding variation. Genome Biol. 12(9), R84 (2011).
161000 Genomes Project Consortium, Abecasis GR, Auton A et al. An integrated map of genetic variation from 1092 human genomes. Nature 491(7422), 56-65 (2012).

17 Ward K, Ogilvie JW, Singleton MV et al. Validation of DNA-based prognostic testing to predict spinal curve progression in adolescent idiopathic scoliosis. Spine (Phila. Pa 1976). 35(25), E1455-E1464 (2010).

18 Ogura Y, Takahashi Y, Kou I et al. A replication study for association of 53 single nucleotide polymorphisms in a scoliosis prognostic test with progression of adolescent idiopathic scoliosis in Japanese. Spine (Phila. Pa 1976). 38(16), 1375-1379 (2013).

19 Xu L, Qin X, Sun W et al. Replication of association between 53 single-nucleotide polymorphisms in a DNAbased diagnostic test and AIS progression in Chinese Han Population. Spine (Phila. Pa 1976). 41(4), 306-310 (2016).

20 Tang QL, Julien C, Eveleigh R et al. A replication study for association of 53 single nucleotide polymorphisms in ScoliScore test with adolescent idiopathic scoliosis in French-Canadian population. Spine (Phila. Pa 1976). 40 (8), 537-543 (2015).

21 Roye BD, Wright ML, Matsumoto $\mathrm{H}$ et al. An independent evaluation of the validity of a DNA-based prognostic test for adolescent idiopathic scoliosis. J. Bone Joint Surg. Am. 97(24), 1994-1998 (2015) 